\title{
Dynamic Radio Configuration of Self-Organizing Base Stations
}

\author{
Henning Sanneck ${ }^{\# 1}$, Yves Bouwen ${ }^{* 2}$, Eddy Troch ${ }^{* 3}$ \\ \# Nokia Siemens Networks GmbH \& Co. KG, Munich, Germany \\ ${ }^{1}$ henning.sanneck@nsn.com \\ *Devoteam NV/SA, Herentals, Belgium \\ $\left\{{ }^{2}\right.$ yves.bouwen, ${ }^{3}$ eddy.troch $\} @$ devoteam.com
}

\begin{abstract}
In current cellular networks the rollout of base stations needs to be conscientiously prepared, because their radio configuration should match with the coverage provided by existing cells. The radio planning for every new cell requires considerable human resources. This paper proposes a framework for Dynamic Radio Configuration (DRC) of future Self-Organizing base stations, which is adaptive to the current network topology context. This function enables the deployment of base stations and new cells in an ad hoc manner, reducing OPEX. An example for DRC of the Physical Cell ID in LTE is presented and the impacts on the LTE OAM architecture are discussed.
\end{abstract}

\section{INTRODUCTION}

The need for data rates (up to $100 \mathrm{Mbit} / \mathrm{s}$ ) and new services for mobile users have set the scene for $3 \mathrm{G}$ Long Term Evolution (LTE). The LTE Evolved Universal Terrestrial Radio Access Network (E-UTRAN) will be characterized by equal or smaller cell sizes than those of predecessor third generation radio access networks. As a consequence thousands of new radio nodes will need to be installed and properly configured in these mobile networks. Hence NGMN Ltd. [1] has identified "Self Configuration" as one of the mechanisms to facilitate deployment and operation for LTE Networks. Selfconfiguration is the process of bringing a new network element or network element parts into service with minimal human operator intervention. The process encompasses several steps illustrated in Fig. 1.

The transport and radio configuration of legacy base stations is completely based on a careful planning which needs to be conducted by the network operator prior to the installation, using a planning toolset. In this paper it is proposed that a subset of the radio parameters would be assigned dynamically, when the base station is powered up for the first time in the field. For this purpose Dynamic Radio Configuration (DRC) is appended to the self-configuration process.

When incrementally building up the network without SelfConfiguration functions one can either perform a labor intensive radio planning step every time a new network element is inserted or perform periodic radio planning updates anticipating the new eNodeB insertions within the next time frame. In the former case the insertion order of the base stations must proceed exactly as planned. In the latter case one needs to accept that the planning is not representative for the operational network at a certain point in time. Without a representative planning the newly inserted base station and its neighbors will not have an optimal configuration matching the current network topology. When using dynamic configuration this trade-off disappears. DRC will configure the new base station / cell and its neighbors in such a way that the best possible coverage and capacity is achieved given the existing intermediate network topology.

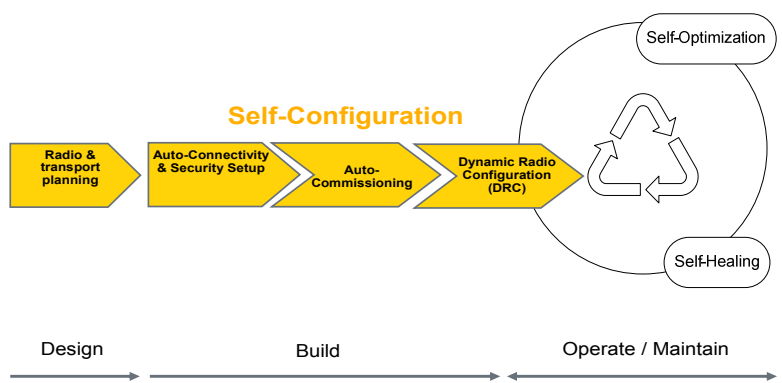

Fig. 1. Scope of the DRC

A second benefit of DRC is a reduction of the planning activities. Although it is still needed to perform a dimensioning and capacity planning for selecting the sites and deciding on the HW resources to be installed, the detailed radio planning can be omitted since the assignment of the corresponding parameters is shifted to the DRC. Where off-line radio planning tools completely rely on input provided by the operator (e.g. expected geographic traffic distribution), DRC algorithms can make use of measurements and already optimized parameters of operational neighbors.

While DRC provides a generic framework, in the following we concentrate on LTE where manufacturers and operators are working together in research [2][3] and standardization to enable the introduction of self-configuration mechanisms.

\section{DRC FUNCTIONALITY}

Radio parameters are classified into two major groups [4]:

1) Class $\boldsymbol{A}$ parameters do not have an influence on the configuration of the adjacent cells or vice versa. Since their 
assignment is independent from the existing network topology, they are out of scope of the DRC.

2) To configure Class $\boldsymbol{B}$ parameters correctly, the knowledge of the relationships and the configuration of adjacent cells is needed (including direct neighbors, neighbors of the neighbors or even all cells in a large geographical area). Since these parameter values are dependent on the current network context, it may be necessary to assign them dynamically. Class B parameters are further divided into subclasses defined by TABLE I:

- Class B1 parameters need to be assigned for an entire network. Hence this class is out of scope for DRC. Examples are Public Land Mobile Network Identity (PLMN ID), frequency band, E-UTRA Absolute Radio Frequency Channel Number (EARFCN) etc.

- Class B2 parameters should be assigned by DRC. Examples are Evolved Global Cell Identity (EGCI) and eNodeB name.

- Class B3 parameters should be assigned dynamically, but not all parameters are needed when an eNodeB is switched on. Examples are Physical Cell Identity (PHY-CID), Physical Random Access Channel (PRACH) root sequence index, Root Sequence Cyclic Shift (RS CS).

- Also class B4 parameters need a dynamic assignment. Examples are initial Neighbor Relationships (NR), quality offset for cell reselection, Tracking Area Code (TAC) and Transmitter Power.

The activity diagram below (Fig. 2) illustrates the individual algorithms executed within DRC, their intermediate outputs and their interdependencies. The algorithms build further on (intermediate) results of previous algorithms. Intermediate results are not directly configuration parameters for the eNodeB, but essential inputs for self-configuration algorithms.
Details on the inputs to the DRC process (equipments parameters, etc. show on the left hand side of Fig. 2) and algorithms for the intermediate outputs of the DRC function (coverage area plan, Pre-operational Neighbor Relation Table (NRT), cell cluster, Class B3 parameters, tracking area) can be found in [3].

TABLE I

CLASS B PARAMETERS

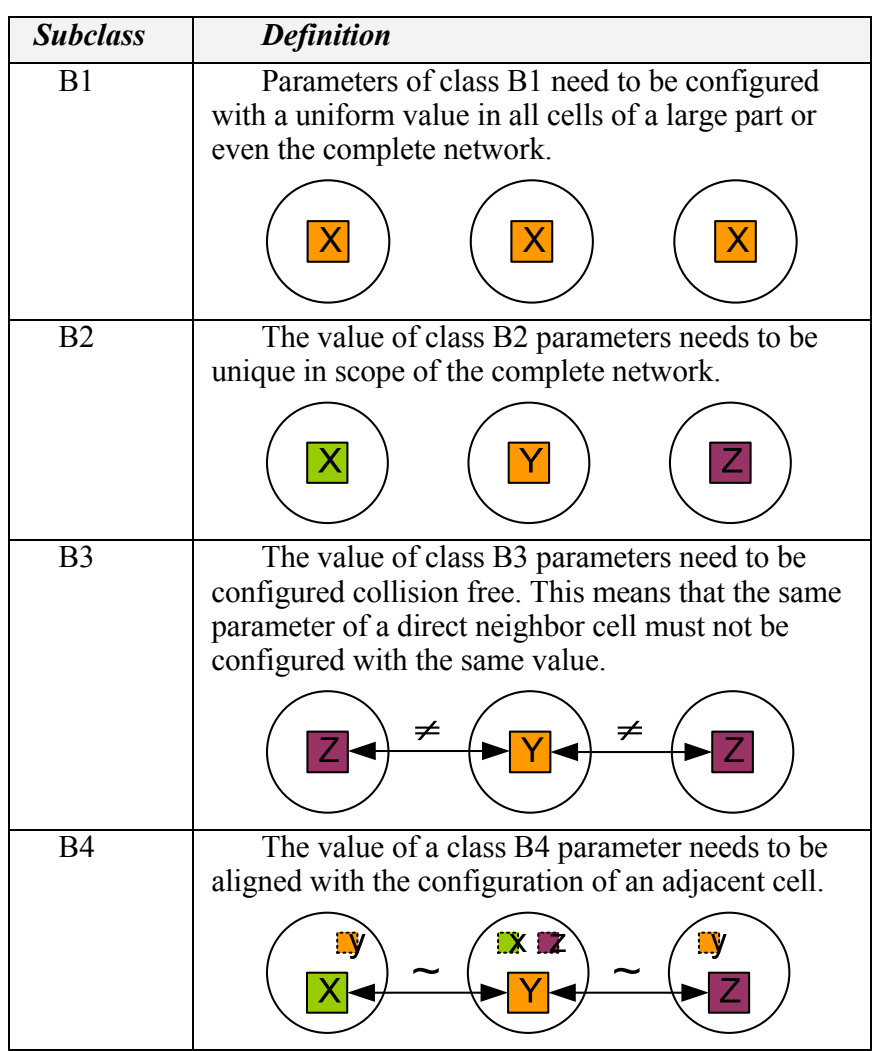

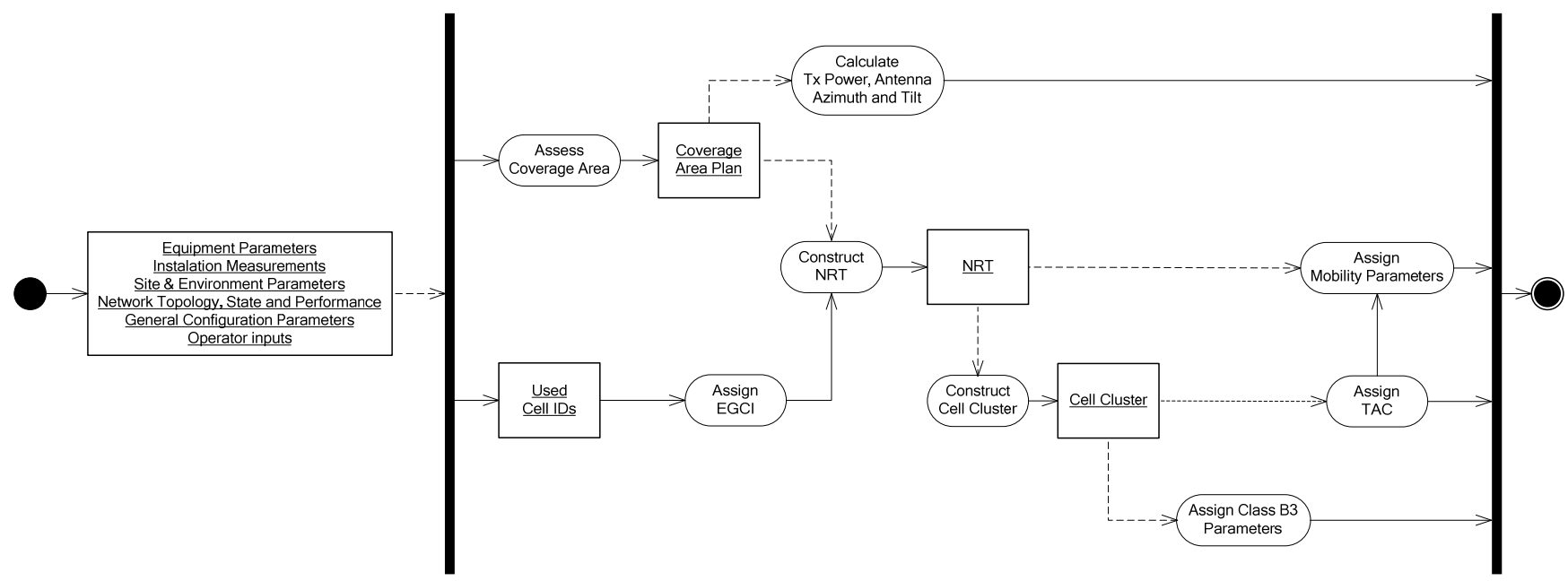

Fig. 2. DRC process and algorithms 


\section{EXAMPLE: LTE PHYSICAL CELL-ID AllocATION}

Class B3 parameters have a restricted value range. Therefore the same value needs to be assigned to multiple cells throughout the network. All class B3 parameters need to be configured collision free (TABLE I), which means that the configured value of the parameter needs to be different from the values configured in all the neighboring cells. An example for a B3 parameter is the Physical Cell ID (PHY-CID) assignment [6]: LTE reference signal sequences, which are comparable to $3 \mathrm{G}$ scrambling codes, are of special interest for self-configuration. They serve as a regionally unique identifier on the physical level because reading and decoding a reference signal takes less than $5 \mathrm{~ms}$. Without an assigned PHY-CID no radio communication is possible within a cell, as the User Equipment (UE) cannot detect the cell. In LTE there are 504 available PHY-CIDs, i.e., IDs need to be reused in a real network deployment.

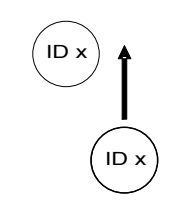
$\begin{array}{ll}\text { a) new cell collides } & \text { b) new cell confuses } \\ \text { with other cell } & \text { other cell }\end{array}$

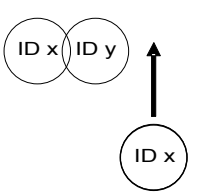

ther cell

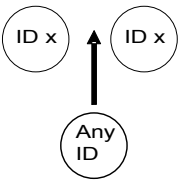

c) new cell gets confused
Fig. 3. PHY-CID Assignment Requirements

Thus, the following requirements for PHY-CID assignments exist (Fig. 3):

- Collision Free: Two neighboring cells may not have the same PHY-CID.

- Confusion Free: A cell may not have two neighbors with identical PHY-CIDs (to enable correct handovers).

Graph coloring is the problem to color the nodes of a graph in a way that two nodes that are connected with an edge are not assigned the same color, with a minimal number of colors. To find a minimal number of colors is known to be a NPComplete problem. From the above requirement definition it is obvious that finding a collision free PHY-CID assignment or a valid coloring for a graph are equivalent problems. To be able to perform the graph coloring, the structure of the cell layout is transformed into a graph with the following steps: Cells are depicted as vertices. The vertices represent neighboring cells in the network are connected by an edge. The resulting graph is then colored. The colors are translated to PHY-CIDs which are subsequently used for Radio Parameter Configuration.

To accommodate the "Confusion Free" requirement the graph is extended with edges to the neighbors of the neighbors which translates the requirement into a problem solvable by graph coloring (Fig. 4). If the colored version of this graph is translated into PHY-CIDs for the cell deployment it is assured that all neighbors of a cell are assigned different PHY-CIDs it is as well collision as confusion free. Note that there is also the special case that a new cell "gets confused" (by becoming a new neighbor to two cells with the same ID) rather than confusing another cell (Fig. 3 c). This case cannot be avoided a priori and requires a specific PHY-CID reassignment algorithm [6].

Existing graph coloring algorithms, however, are suitable only to compute PHY-CID assignments for entire networks rather than incrementally assigning PHY-CIDs for individual nodes. In the latter case they would introduce too much overhead, as for each new cell all cells in a cluster could be affected. Therefore to enable PHY-CID assignment according to the Dynamic Radio Configuration principles outlined in the previous sections a new iterative process which restricts the changes to small parts of the network has to be defined that works on small partitions of the graph, while still retaining the properties of the colored graph, e.g., the minimal usage of colors.
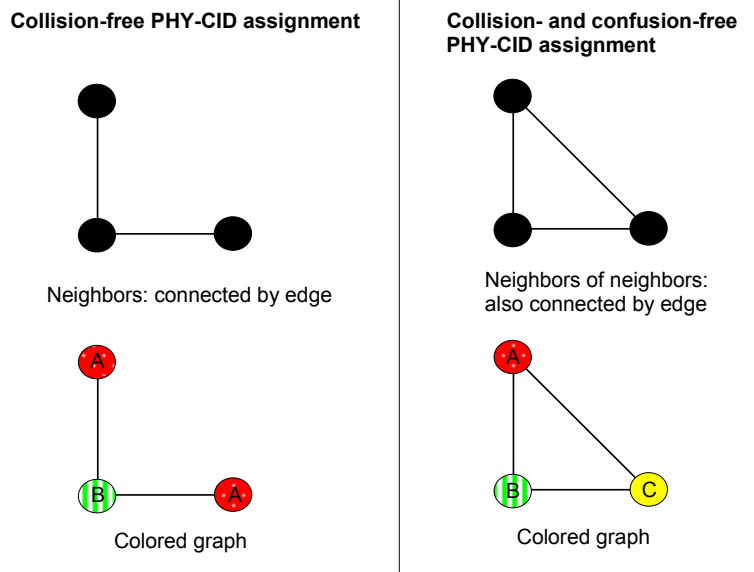

Fig. 4. Mapping PHY-CID assignment requirements to graph coloring

PHY-CIDs are grouped into ID Sets. The following ID Sets are defined to facilitate the description of the algorithm:

- NSet: The PHY-CIDs of the direct neighbors of the new cell

- NNSet: The PHY-CIDs of the neighbors of the NSet

- IDSet-u: All used PHY-CIDs in the network

- IDSet-a: All PHY-CIDs that are available for the network

The sub graph that is considered contains the new cells as center and its NSet and NNSet. It has to be assured that the PHY-CIDs of the nodes in the graph are not changed by another process until all steps have been finished.

The following steps describe the algorithm:

1. Check if the IDSet-u contains IDs that have not been used in the NSet or NNSet of the new cell: result $=I D S e t-u \mid\{N S e t$ $\cup N N$ Set?

\subsection{If the result set is nonempty select the PHY-CID from the result set}

\subsection{If the result set is empty, continue}




\section{If IDSet-a and IDset-u are identical try a re-planning of the complete network}

\subsection{Otherwise introduce a new PHY ID by choosing the lowest PHY-CID from IDSet-a $\mid$ IDSet-u}

Evaluation in [6] has shown that for a first estimation of the required number of PHY-CIDs the well-known coloring algorithm of Powell and Welsh gives a worst case assumption. But in all cases the number of required PHY-CIDs was very close to the maximum number of first-degree neighbors of a single cell within the cluster. This means that even if the degree of the graph is larger than the number of available PHY-CIDs, a collision and confusion free assignment for a specific cell is possible, as long as the maximum number of first-degree neighbors for that cell is less than the number of available PHY-CIDs.

\section{ARCHITECTURE}

\section{A. Architecture options}

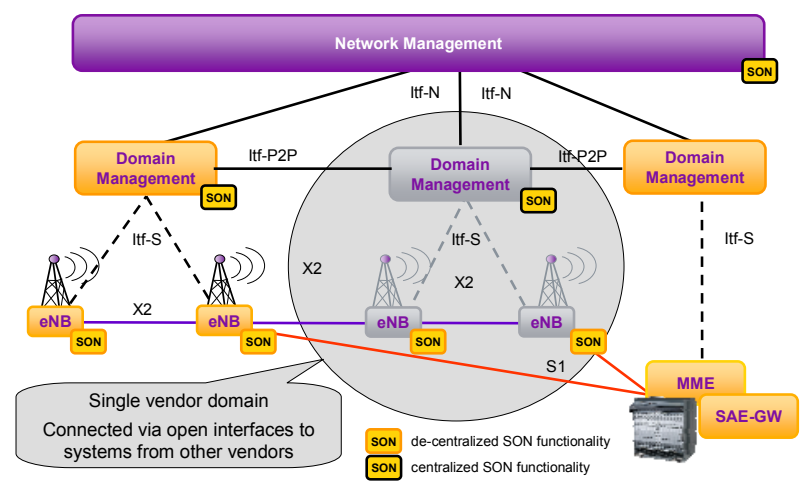

g. 5. Management Reference Model

$\mathrm{Fi}$

Fig. 5 shows the Management Reference Model for EUTRAN. There are several alternatives for locating the DRFC algorithms and their coordination functions:

\section{1) Network management system centric:}

In this architectural option the self-configuration algorithm execution and coordination is situated on the Network Management System (NMS). So doing the algorithm execution and coordination will run on a central entity. The main benefit is that the impacts on interfaces and standardization are relatively limited, since the existing interfaces and configuration management functions of the layers underneath can be re-used. The solution can also be deployed on an installed base of eNodeBs without any retrofitting. The main technical challenge in this architecture is the integration of the data from the different new input sources to fulfill the selfconfiguration process. The data can come from online traffic models, test probes, eNodeB inventory data, etc.

\section{2) Domain manager centric, with Itf-P2P:}

In this architecture the SON algorithm execution and coordination is distributed among the Domain Managers (DM) of the different vendor domains. The information exchange and coordination between DM's is done on the Peer-to-Peer Interface (Itf-P2P). Although the SON algorithm execution and coordination run distributed on the different domain managers, some central component on the NMS level is defined for human control and monitoring. The DM where the new eNodeB is inserted will temporarily take the role as master and will be able to trigger and monitor a reconfiguration of nodes in another vendor domain via the Itf-P2P. Here the impact on the interfaces and the standardization are clearly very high. The scope of the Northbound interface (Itf-N) now moves from managing the eNodeB to managing the self-configuration process itself. Via Itf-N the self-configuration process will be monitored and controlled. Until now the Itf-P2P is merely a theoretical concept in $3 \mathrm{GPP}$. In practice when one wants to utilize this interface one will introduce a new interface in the architecture.

\section{3) Domain manager centric, without Itf-P2P.}

This architecture is similar to the previous one but here the Itf-P2P is not used. In order to mimic the function of the ItfP2P, the NMS would act as a relay and proxy function. Relay in case reconfiguration needs to be triggered and proxy in case of data exchange. The big advantage of this architecture option is that there is no need to set up the Itf-P2P. On the other hand the impacts are moved to the Itf-N and one needs to able to rely on the fact that the NMS system will provide the needed relay and proxy function. The relay point on the NMS level introduces additional complexity, for example in terms of coordination and exception handling.

\section{4) eNodeB Centric:}

The algorithm execution and the DRC process coordination is situated on the eNodeB. The algorithms could run completely distributed, but to simplify the data acquisition, computation and coordination so called localized algorithms could be used. In the execution of a localized algorithm only a few nodes will participate which are geographically close to each other. On the NMS level similar impacts as the for the "DM centric without Itf-P2P" solution are needed. In addition the southbound interface needs to support monitoring, control and input data provisioning for the algorithms. The newly inserted eNodeB should be able to autonomously discover all neighboring eNodeBs to set up the X2 interfaces. This could for example be done by using a beacon signal, yet this would affect the LTE radio interface standards and increase the eNodeB hardware cost. Localized algorithms would also require extensive data exchange and coordination over the X2 interface. It must be assured at all times that the input parameters are synchronized between the eNodeBs and aligned with the corresponding central management functions. All existing eNodeBs must have been upgraded to support the required functionality, which is not obviously given in a multivendor network. 


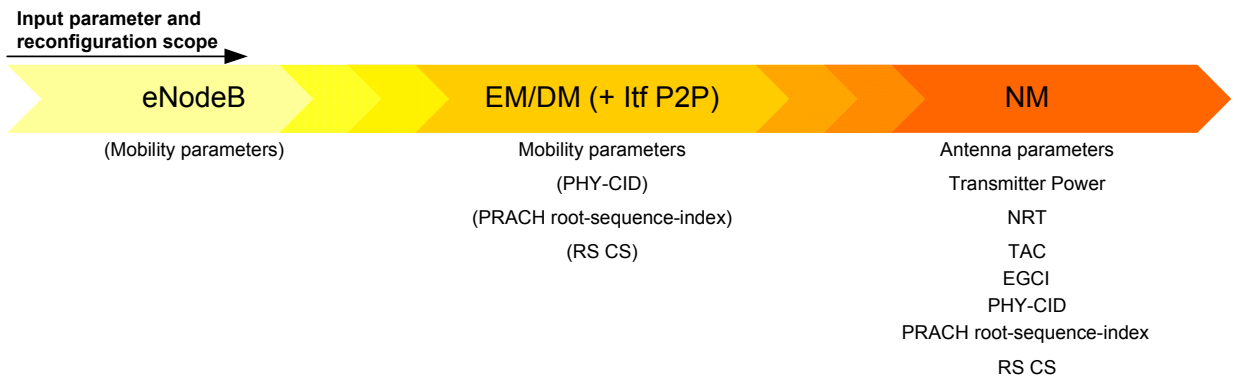

Fig. 6. Preferable location of DRC algorithms

\section{5) Hybrid solutions:}

In hybrid solutions the algorithms themselves and the DRC process coordination function is partitioned in such a way that one part is done on one level and another part on another level. Such a partitioning can be among the NMS and DM, the NMS and eNodeB (where the DM act as mediator), the eNodeB and DM or even among all levels in the reference architecture eNodeB, DM and NMS. The main pitfall of hybrid solutions is that a careful coordination and synchronization between the different levels will be needed. Given that the different levels could act as a Policy Enforcement Point (PEP) their configured policies may result in conflicts between the levels

\section{B. Preferable architecture}

In principle the preferred architecture may be different for each parameter to be configured. Fig. 6 illustrates which architecture should be used for which parameter. For simplicity of the coordination functions, the larger the geographical scope of the input parameters and of the existing cells that may need to be reconfigured, the higher the management level where the algorithm and coordination function should be located. The algorithms for most of the parameters should therefore reside at network management level. The parameters in brackets indicate a secondary, less preferable location.

In general following characteristics of the DRC algorithms can be observed:

- input parameters: required with a multiple cell scope and even a multi-vendor domain scope; need to be collected from different sources and be integrated

- coordination: in a chain of sequential functions; between multiple NE: reconfiguration of already operational cells and the move to the operational state of new cells

- limited scalability requirement: the algorithms are executed at eNodeB or cell insertion time (but for thousands of nodes) and only relatively rarely reexecuted for operational nodes

- the performance with respect to response time is not that critical, due to the execution done mainly during pre-operational (build) phase
Given those characteristics, a centralized execution and coordination of the algorithms is sufficient and easier to realize than an eNodeB centric solution.

\section{CONCLUSIONS}

A framework is presented for a dynamic radio configuration of new base stations or cells, allowing for ad-hoc deployments in cellular networks with minimal planning overhead. These functions primarily consist of a set of algorithms to compute the radio parameters automatically and coordination functions to collect the necessary input parameters, to configure the new cells and to reconfigure neighbor cells. Altogether these functions are activated in a (usually centralized) workflow according to a predefined order while taking into account operator policies. Algorithms can be derived from those available in the conventional network planning \& configuration tool chain, yet have to be adapted to the dynamic (incremental) way of execution. The concept enables a transition path from a "static" infrastructure network where all parameters are pre-planned before deployment to an entirely "dynamic" infrastructure network where only the site of deployment for a network element is pre-planned.

\section{REFERENCES}

[1] Next Generation Mobile Networks, NGMN Recommendation on SON and O\&M Requirements, December 2008. http://www.ngmn.org/uploads/media/NGMN_Recommendation_on_SO N_and_O_M_Requirements.pdf

[2] SOCRATES, Self-optimisation and self-configuration in wireless networks, European Research Project, http://www.fp7-socrates.eu.org.

[3] Henning Sanneck, Yves Bouwen, and Eddy Troch, Context based configuration management of plug \& play LTE base stations IEEE/IFIP Network Operations and Management Symposium, Osaka, Japan, April 2010.

[4] 3GPP TSG-SA5 contribution S5-091879, Nokia Siemens Networks, "Starting material for automatic radio network configuration data preparation".

[5] 3GPP, Evolved Universal Terrestrial Radio Access (E-UTRA) and Evolved Universal Terrestrial Radio Access Network (E-UTRAN), Overall Description, Stage 2, TS36.300, http://www.3gpp.org

[6] Tobias Bandh, Georg Carle and Henning Sanneck, "Graph coloring based physical cell ID assignment for LTE networks", IWCMC 2009. 